\title{
Analog and semantic models of judgments about the months of the year
}

\author{
WILLIAM J. FRIEDMAN \\ Oberlin College, Oberlin, Ohio
}

\begin{abstract}
This study tested undergraduates' ability to judge cyclic relations between the months of the year, days of the week, and buildings around a campus square. On each trial, a pair of month, day, or building names was presented. Subjects judged whether the second stimulus was closer to the first going forward or backward in time (or clockwise/counterclockwise around the square). For all three contents, response times and errors increased as the second stimulus ap. proached the direction boundary. The results can be explained by the types of analog models used to account for the symbolic distance effect for bipolar continua. In contrast, semantic models of the symbolic distance effect appear to be poorly suited for explaining how cyclic comparisons are made.
\end{abstract}

Psychological research methods used over the last century and specific work in cognitive psychology over the last decade make it abundantly clear that humans can make principled comparative judgments on a wide variety of dimensions. Far from clear, however, is how the information underlying comparative judgments is coded and exploited. Most of the work on this issue has surrounded what is called the symbolic distance effect (SDE; Moyer, 1973). The SDE is the tendency for subjects to take increasing time to compare two symbols as they become closer in value on the dimension of interest. The SDE is quite robust, having been demonstrated with very heterogeneous content (see Banks, 1977, Holyoak, 1978, and Shepard \& Podgorny, 1978, for summaries of research and a more thorough description of the theories). Several distinct processing models have been proposed to explain the SDE, of which analog and semantic models are the most influential. According to analog models, an integrated representational continuum encodes the two symbol magnitudes. The comparison operation functionally resembles the overt discrimination between two visible stimuli on a dimension such as size or proximity to a reference point. The SDE is explained by the assumption that similar values on the representational continuum are more difficult to discriminate. In the opposing semantic models, magnitude information is encoded for each symbol in a discrete linguistic format. Comparisons are made by reading information from the two sources and determining which better matches the criterion. When the two sources

This work was supported by a Research and Development grant from Oberlin College. Thanks are extended to Lee Fertig and David DuBois for testing the subjects. Requests for reprints should be sent to William J. Friedman, Department of Psychology, Oberlin College, Oberlin, Ohio 44074. differ only slightly, they are likely to receive the same initial code and the matching process must wait for additional information.

Both classes of models offer plausible accounts of the processes involved in the comparison of two symbols on a bipolar continuum. On a given continuum, two values can be characterized as differing either in their absolute magnitude, as is emphasized by the semantic models, or by their relative magnitude, as is emphasized by the analog models. However, in the special case of cyclic continua, the two types of descriptions diverge in their ability to capture relationships. Consider a hypothetical continuum in which the numbers 1 through 10 form a repeating cycle, with each number equidistant from its neighbors on the dimension of interest. An integrated representation that encodes position-like information, analogous to a circle of numbers, captures a variety of relationships that cannot easily be encoded in discrete descriptions of the individual numbers. Examples include the relationships of bidirectional proximity ( 8 is closer to 1 than it is to 3 on the dimension) and opposition $(1-6,2-7 \ldots 10-5$, all form pairs farthest apart on the dimension). A semantic account would require that the individual descriptions of 8,3 , and 1 could lead to the extraction of the opposition of 8 and 3 and the greater proximity of 8 to 1 than to 3. Short of coding for each value the degree of proximity to each other value, it is not clear how a semantic model would accomplish this.

The importance of cyclic continua for theories of symbolic comparisons was previously noted by Te Linde and Paivio (1979), who provided strong evidence for proximity effects with the hue circle. Subjects were presented with a color name and then two color chips. Their task was to decide which of the two chips was more similar in color to the color name. The results showed that responses were 
quicker the more the two chips differed in their subjective hue distances from the color name. The demonstration of proximity effects for the hue circle is troublesome for semantic models on two grounds. First, as Te Linde and Paivio (1979, p. 142) point out, knowledge about relations between colors is probably mediated by imagery rather than linguistic representations. Second, hues are formally cyclic, and cyclic relations are not easily described by semantic codes (Te Linde \& Paivio, 1979, p. 147). However, it could be argued that comparing remembered colors is functionally similar to making perceptual judgments, but that for most other symbolic contents (e.g., number magnitude, animal sizes) proximity effects are attributable to properties of their semantic representations. Given the special representational properties of colors, it is important to show cyclic proximity effects for contents that semantic theorists would predict are coded linguistically.

In the present study, we selected two familiar cyclic continua, months of the year and days of the week. These have formal properties similar to the hue circle or the number cycle described above. For example, it is reasonable to ask if October is closer to February or May or to describe month pairs that are farthest apart in time. One of these systems, the months of the year, has been used previously in symbolic comparison tasks (Fairbanks, 1969; Friedman, 1983; Seymour, 1980a, 1980b) and has been the subject of analog (Friedman, 1983) and semantic (Seymour, 1980a, 1980b) models.

The previous studies showed clear proximity effects, but most have treated the months as a linear continuum and do not consider cyclic relationships. Fairbanks (1969) asked subjects to determine which of two months was closer to the end of the year. He found that response time was a decreasing monotonic function of the degree of separation of the two stimuli. In one experiment, Seymour (1980b) had subjects judge whether a month fell in the first half (JanuaryJune) or second half (July-December) of the year. Response times were longest for months close to the June-July boundary. In two other experiments (Seymour, 1980a, 1980b) "central months" (AprilSeptember) were discriminated from "end months" (January-March and October-December). Again, the longest response times occurred adjacent to category boundaries, in this case around March-April and September-October. None of the experiments tested cyclic relationships; first-half/second-half and central-end categories can easily be defined for a January to December linear continuum.

Friedman's (1983) Experiment 2 used a cyclic relationship of the months and demonstrated an interval-comparison effect, an effect related to the SDE and other proximity effects according to an analog model. Subjects compared the length of two intervals of months, and the intervals were free to cross the December-January boundary. An example of an item is determining which is shorter, November to February or April to August. It was found that response times were greatest when the two intervals were most similar in length. The results were interpreted as supporting an analog process, but there is some question as to whether a semantic model could also account for the interval-comparison effect. It is possible to argue that problems with a greater difference in interval length are more likely to differ in the number of month categories spanned. Such categories might be similar to Seymour's (1980b, p. 392) "locative codes" (e.g., "start, end, early, late, central, peripheral") or some sort of seasonal grouping. Several such models do not produce sufficiently high levels of accuracy when applied to sample problems (Friedman, 1983, Table 2), but it is possible that the right categories remain to be tested.

In the present study, subjects were presented one month name and then another and asked to determine whether the second month was closer to the first going forward or backward in time. For example, for the pair April-November, the correct answer would be backward, whereas for the pair July-November, the correct answer would be forward. All month pairs except those 6 months apart were used. Successful performance on this task depends on exploiting cyclic relationships, not linear relationships, between the months or fixed categories.

The following analog model, adapted from Friedman (1983), was used to predict performance on this task. In the earlier paper, I suggested that image processes, of the sort described by Kosslyn (1981) and Paivio (1978), are involved in judgments about the relative proximity of months of the year. The process included an image-generating mechanism and a set of operations that can be performed on the image once constructed. The images are assumed to be analogous to schematizations of the cycle of months, such as linear or circular diagrams. Among the set of operations are ones analogous to activating and detecting the relative positions of points on a diagram. The assumed analog for the present cyclic direction task is a perceptual task in which two points on the perimeter of a circle are illuminated in succession and subjects determine whether the second point is closer to the first going clockwise or counterclockwise. In the perceptual task, one would expect that separations near $180 \mathrm{deg}$ would be the most difficult to judge and, as for most psychophysical discriminations, that difficulty would increase progressively as the dividing line was approached. In the present month task, I assume processes akin to activating the position of the 1st month, then determining and activating the approximate boundary region 6 months away, and finally activating the position of the 2 nd month and comparing it to the other two. It is assumed, by 
analogy to the perceptual task, that the comparison process will progressively increase in difficulty as the second month approaches the boundary region. This would result in both linear and quadratic effects for response time and/or number of errors as a function of cyclic distance between the pair of stimulus months. In addition, the simultaneous activation of multiple "positions" in the image representation should make the backward problems as easy as forward problems.

It is difficult to derive an alternative semantic model for the task from existing semantic models. For example, a semantic model of digit magnitude comparisons (Banks, 1977; Banks, Fujii, \& Kayra-Stuart, 1976) incorporates a number of features that are restricted to bipolar continua and bipolar comparisons. In this model, whatever the representational base, "the processing codes are assumed to be the discrete, componential codes of natural language" (Banks, 1977, p. 131). In the first of two stages, stimulus digits are coded as larger than or smaller than a randomly varying cutoff point. In the second stage, these codes are compared with "instructional codes" that are in the same format. The following questions illustrate some of the problems in applying this model to the cyclic direction judgment task. Given the changing reference point, how would the cutoff point be described in the model? How are codes like "forward" or "backward" generated in the first stage, or, alternatively, how are instructional codes made comparable to stage 1 output? If these are natural language codes, how can they incorporate the necessary cyclic relational information?

Seymour (1980a, 1980b) has extended the Banks et al. (1976) model to account for the results of some of his month tasks. He suggests that month order is coded, in part, categorically, using such codes as "first," "early," "central," "late," and "last." The model can explain the difficulty subjects have in classifying months near category boundaries but is not readily applicable to the cyclic direction task. There is no obvious way to match these codes with the criteria: closer backward or forward. Even if one supplemented categorical knowledge with a set of decision rules (e.g., "if the first month is 'early' and the second month is 'late,' say 'backwards." "), fundamental problems would remain. For example, March and April are both "early" months in one of Seymour's (1980a, p. 261) illustrations, whereas September and October are both "late" months. In the cyclic direction task, however, October is closer to March going backward from March, whereas September is closer to April going forward from April. Seymour's backup process for making fine order discriminations, context sensitive coding (Wickelgren, 1979), does not help here because it does not convey information about separations of nonadjacent months. Other sorts of problems would ensue whenever the two stimulus months fell in the same category or whenever one or both fell near category boundaries.

Simple arithmetic models based on a conversion of month names to the numbers 1 through 12 are also inadequate for the task. Neither relative magnitude nor magnitude of difference is sufficient to make cyclic direction judgments. Four decision rules coupling magnitude and difference (e.g., "if the first month is less than the second month and their difference is greater than 6, say 'backwards" ") could lead to correct solutions. However, the decision rules alone do not predict longer response times for differences near 6. Even if one assumes some sort of proximity effect for comparing differences to the value 6 , one would expect similar proximity effects for comparing the magnitude of the two stimuli. This would lead to the longest response times for separations near 1 and 6 and thus no linear separation effect. In addition, if subtraction is a component process, one would expect that the smaller the absolute value of the difference between month numbers and the less the magnitude of the smaller number, the faster would be the response (Shoben, Čech, \& Schwanenflugel, 1983). For the month problems used in the present study, cyclic distance (number of months' separation in the closer direction) is only weakly or moderately correlated with either of these variables. Thus, it is possible to test whether the subtraction variables or the analog variables better predict performance variation across problems.

Serial activation models also deserve consideration, because months are experienced in an invariant order and subjects often report reciting portions of the month order (Friedman, 1983). A number of studies of alphabet- or month-order judgments show approximately linear separation effects, usually about 250-500 msec for each intervening item, and a strong advantage of forward- over backward-order tasks (e.g., Friedman, 1983; Hamilton \& Sanford, 1978; Lovelace, Powell, \& Brooks, 1973; Seymour, 1980a, Experiment III; Weber, Cross, \& Carlton, 1968). Serial activation of the month names could be used to solve cyclic direction problems if subjects counted each step between the first and second stimulus months up to a maximum of 5 steps. Presumably, subjects would use the forward order and terminate the search once either the 5th month in order or the 2nd stimulus month was encountered. This would lead to a linear increase in response time from 1- to 5 month forward separations and a flat backward curve with latencies longer than for 5 months forward. These predictions differ from the analog model's in the linearity of the forward curve and the asymmetry of the forward and backward curves.

Two experiments were conducted to demonstrate the proximity effect for cyclic relationships. In Experiment 1 , subjects made direction judgments for all possible pairs of month names and also performed a 
similar spatial task. The spatial task required direction judgments for pairs of buildings drawn from 12 buildings located around a campus square. This task was chosen as a model task because many researchers believe that spatial representation involves analog properties. In Experiment 2, the direction judgment method was applied to another cycle, the days of the week, in an effort to replicate the findings of Experiment 1 .

\section{EXPERIMENT 1}

\section{Method}

Subjects. Twelve undergraduates, half male and half female, served in the experiment. They were paid $\$ 3$ for their participation.

Stimull and Procedure. Each subject was tested in a temporal condition and a spatial condition, with half receiving each order of the two. The subjects were tested individually in single sessions in approximately $40 \mathrm{~min}$. Stimuli for the temporal condition were the 120 possible pairs of month names that exclude duplicates and pairs 6 months apart. The stimuli for the spatial condition were drawn from a set of 12 familiar buildings that are spaced about equally, 3 to a side, around the main campus square of Oberlin College. Again, 120 pairs were used, excluding duplicates and pairs 6 buildings apart. Stimulus pairs were presented in a different random order for each subject and condition. In the temporal condition, the subjects received the following instructions: "In this experiment you will receive a series of problems concerning the months of the year. For each problem one month name will be displayed and moments later another month name will be displayed. We would like you to judge whether the second month is closer to the first going forward or backward in time. If the second month is closer going forward in time you should press the " $/$ ' key. If it is closer going backward in time you should press the ' $Z$ ' key. You will control when each problem begins. When you are ready, press any key. Once a problem has been presented, try to respond rapidly but accurately." In the spatial condition, "building" was substituted for "month" and "clockwise" and "counterclockwise" for "forward" and "backward," respectively.

Instructions and stimuli were presented on a Zenith Model ZVM-121 video monitor, and response time and correctness were recorded from the keyboard of an Apple II Plus computer, which controlled the experiment. Stimuli were capital-letter month or building names centered horizontally and vertically on the screen. The first stimulus appeared for 2 sec, followed by a blank screen for $.5 \mathrm{sec}$ and then stimulus 2 . Stimulus 2 remained on the screen until the subject responded, with a maximum response time of $9.9 \mathrm{sec}$.

Following each condition, the subjects completed a written method-report sheet adapted from Paivio (1975). They were asked to rate the importance of a number of possible methods of solving the problems using a 7-point scale, with 1 labeled "never used" and 7 labeled "used on almost all problems." For the temporal condition, the methods were : (1) using a logical rule, (2) picturing the months in one's mind, (3) counting on one's fingers, (4) saying the intervening months to oneself, (5) using month numbers, and (6) using associated feelings or colors. For the spatial condition, the methods were (1) using a logical rule, (2) picturing in one's mind, (3) counting on one's fingers, (4) saying the intervening buildings to oneself, and (5) using associated feelings or colors.

Design. Stimulus pairs were categorized according to the relationship between the two stimuli in terms of direction (closer forward/backward or clockwise/counterclockwise) and distance $(1,2,3,4$, or 5 months or buildings) in that direction. There were 12 replications, corresponding to the 12 possible stimulus 1 elements, for each direction-distance combination. Task order and sex were between-subject factors, and condition (temporal vs. spatial), direction, and distance were within-subject factors.

\section{Results}

Figure 1 presents the response times for correct trials for the temporal and spatial conditions as a function of direction and distance. As predicted, response times were greatest for problems in which stimulus 2 was close to the direction dividing line, and this was true for both the temporal and spatial conditions. Differences between the means were tested by a repeated measures ANOVA with task order, sex, condition, direction, and distance as factors. Task order and sex were not significant as main effects or in interactions. The effect of principle interest, distance, was highly significant $[F(4,32)=$ $40.29, p<.0001]$. The distance effect interacted weakly with condition $[F(4,32)=3.01, p<.05]$, apparently reflecting more quadratic spatial curves. A weak direction effect $[F(1,8)=8.38, p<.05]$ revealed that forward/clockwise problems were solved more quickly than backward/counterclockwise problems. Direction did not interact with condition. Finally, a weak direction $\times$ distance interaction $[F(4,32)=2.72$, $\mathrm{p}<.05]$ seems to reflect a more quadratic backward/ counterclockwise curve. The three-way interaction with task was nonsignificant.

Linear and quadratic components of the distance effect were tested by planned comparisons. In the temporal condition, both linear $[\mathrm{t}(32)=11.65, \mathrm{p}<$ $.001]$ and quadratic $[\mathrm{t}(32)=3.25, \mathrm{p}<.01]$ trends were significant. The spatial condition also showed significant linear $[\mathrm{t}(32)=8.76, \mathrm{p}<.001]$ and quadratic $[\mathrm{t}(32)=6.21, \mathrm{p}<.001]$ trends. The quadratic trends reflect an accelerating distance effect as the dividing line is approached.

Regression analysis was used to test whether cyclic distance or a pair of variables reflecting a possible subtraction process beter predicted problem varia-

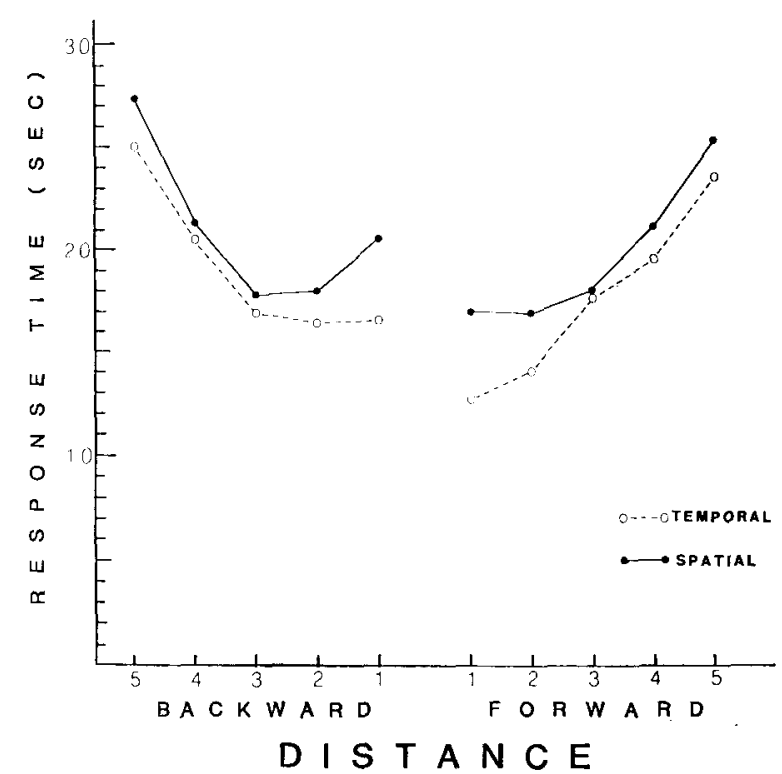

Figure 1. Mean response time (in seconds) for each dhatance and direction for the temporal and spatial taks in Experiment 1. 
tion in response time. Mean response time, including both correct and incorrect trials, for the 120 problems was regressed on distance from the dividing line in one analysis and on a linear combination of difference between month numbers and the magnitude of the smaller number for the problems in another analysis. As previous analyses revealed, the linear component of distance from the dividing line was highly related to a problem's mean response time $(R=.77)$. The two subtraction variables provided a substantially poorer fit $(R=.49)$. When cyclic distance was entered into a multiple regression as the first predictor, neither of the subtraction variables accounted for more than $2 \%$ additional variance. Apparently, the relation between the subtraction variables and response time is mediated by the correlation between numerical difference and cyclic distance (Pearson $r=.53$ ).

Figure 2 shows the mean number of errors out of a possible 12 as a function of condition, direction, and distance. Again, problems of distance \pm 5 were the most difficult. Number of errors was tested by an ANOVA identical to that for response time. Neither of the between-subjects variables was significant. Distance was highly significant $[F(4,32)=23.50$, $p<.0001]$. The only other significant effect was the interaction between distance and condition $[F(4,32)$ $=4.53, \mathrm{p}<.01]$. This appears to reflect more quadratic temporal curves, a finding that offsets the more quadratic spatial curves for response time.

The distance effects were partitioned into linear and quadratic components by separate sets of planned comparisons for each condition. For the temporal condition, both linear $[\mathrm{t}(32)=7.10, \mathrm{p}<$ $.001]$ and quadratic $[t(32)=3.46, p<.01]$ trends were

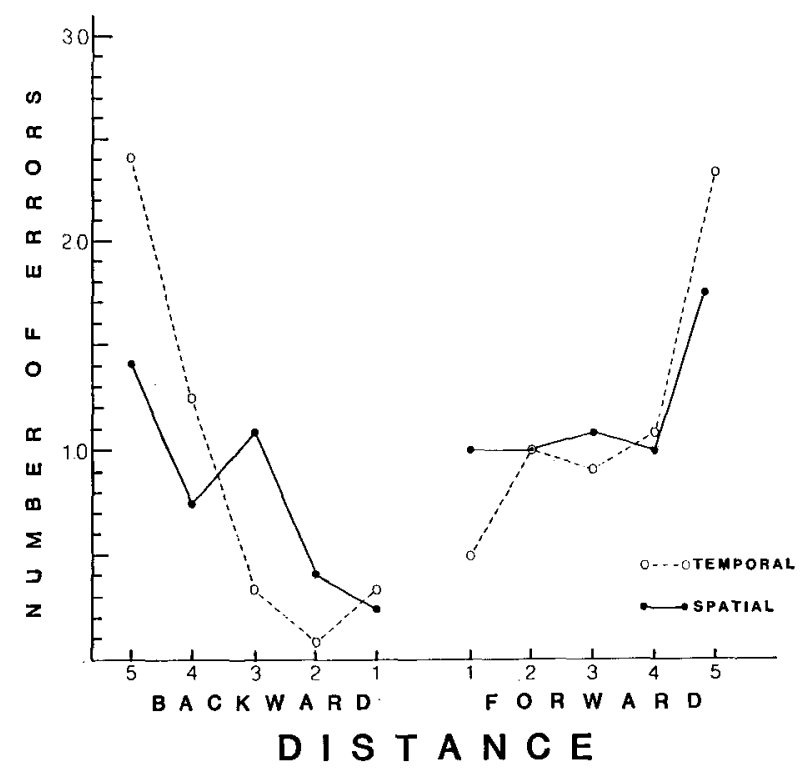

Figare 2. Mean number of errors ont of a posalble 12 for each distance and direction for the temporal and apatial taks in Experment 1. significant. Only the linear trend was significant in the spatial condition $[t(32)=3.28, p<.01]$.

Method importance ratings were analyzed by separate repeated measures ANOVAs for the spatial and temporal tasks, with method as a within-subject factor. For the temporal task, the method means were: (1) 3.75 , (2) 4.21, (3) 1.29 , (4) 2.63 , (5) 4.12 , and (6) 2.88. Picturing the months is the strongest reported method, followed closely by using month numbers. The third most frequent category, using a logical rule, is unfortunately ambiguous, and the fact that it is even reported in the spatial task (see below) probably justifies disregarding it. The ANOVA showed a weak method effect $[F(5,55)=2.76, p<$ $.05]$. In the spatial task, the subjects strongly and uniformly reported imagery. The method means were: (1) 3.42, (2) 6.87, (3) 1.04, (4) 2.88 , and (5) 2.46 , and the method effect was highly significant $[F(4,44)=19.29, p<.0001]$.

\section{Discussion}

The response time curves fit the analog model well and are similar for the temporal and spatial content. The linear effects are inconsistent with the hybrid arithmetic-decision rule model, and the quadratic effects and forward-backward symmetry are inconsistent with the serial activation model. With the exception of the forward temporal curve, there is little increment in response time up to a distance of 3 months or buildings, but the time increases from about 600 to $950 \mathrm{msec}$ from distances of 3 to 5 months or buildings. The means for temporal forward 1 and 2 months were slightly lower than one would expect from the rest of the curve. Since the months are overlearned as a verbal list (Friedman, 1982, 1983), it seems reasonable to suppose that when the second stimulus month closely follows the first in list order, part of the list representation is automatically activated. Presumably, for these types of problems, subjects exploit the verbal list information rather than use an analog process.

The error data showed a pattern similar to that for response time, although with the greater instability that one would expect with dichotomous data. Error rates were low and fairly flat for distances of 1 to 3 or 4 , with a subsequent doubling of number of errors.

If one disregards the means for temporal forward 1 and 2 months, then the direction effect in response time appears to stem exclusively from the spatial condition. Since there is no reason why clockwise judgments should be easier than counterclockwise judgments and since there was no direction effect for accuracy, it seems reasonable to conclude that the direction effects are attributable to chance. The absence of direction effects would be consonant with an analog model and would seem to rule out the serial activation model. Conclusions about the pres- 
ence or absence of direction effects must be qualified by the caution that handedness of responses is confounded with direction.

The introspective data support the use of imagery in the spatial condition and, less clearly, of imagery and month numbers in the temporal condition. While reports of the use of month numbers in the temporal condition might seem to favor an arithmetic model, arithmetic operations alone are insufficient for solving the task and, as noted, the linear distance effect seems inconsistent with a hybrid subtraction-decision rule model. It may be that subjects sometimes use addition or subtraction to determine the month 6 months away from the first month but nonetheless resort to an analog process to determine the relative temporal location of the second month. However, there is no independent support for arithmetic operations in the regression analyses.

The results of Experiment 1 indicate that a process with analog properties is involved in judging a cyclic relationship of the months of the year. The findings are consonant with those of Friedman (1983) and are not easily explained by semantic models of knowledge of the months. Experiment 2 tested the generality of the same proximity effect with a new content, the days of the week.

\section{EXPERIMENT 2}

In this study, subjects were presented with each nonduplicating pair of the days of the week and asked to judge whether the second day was closer to the first going forward or backward in time. The principle difference in the procedure was that the stimuli were presented auditorily and the subjects responded vocally. The use of vocal responses guards against the possibility that the left-hand/right-hand dichotomization of past and future is responsible for the use of analog representations.

\section{Method}

Subjects. Nine female and eight male undergraduates served as subjects. They were not paid for participation.

Stimall and Procedure. The subjects were individually tested in single sessions of less than $30 \mathrm{~min}$. The stimuli were the 42 nonduplicating pairs of days of the week. Each subject received the 42 pairs in a different random order. The subjects were read the following instructions: "For each of the following problems I'm going to give you one day of the week and then another. I want you to tell me whether the second is closer to the first by going forward in time from the first or backward in time from the first. For example, I will say 'A ... (brief pause) B.' If B is closer going forward in time from A, then you would say 'after.' If B is closer going backward from $A$, then you would say 'before.' " On each trial, the experimenter read the pair of names aloud separated by a brief pause. Response time was recorded by stopwatch from the end of the articulation of the second day to the beginning of the response. First responses were recorded and later scored as correct or incorrect. At the end of the testing, subjects filled out a method report sheet identical to the one used in the month condition of Experiment 1, with the exception that the word "day" replaced the word "month."

\section{Results and Discussion}

Figure 3 presents the mean response times for correct trials as a function of distance and direction. As in Experiment 1, the longest responses occurred adjacent to the forward-backward boundary. Response times were subjected to a repeated measures ANOVA with sex as a between-subject factor and direction and distance as crossed within-subject factors. Sex was not significant as a main effect or in interaction. Distance was highly significant $[F(2,30)$ $=31.45, \mathrm{p}<.0001]$. Direction was also significant $[F(1,15)=10.19, p<.01]$, but its interaction with distance was not. Planned comparisons for the distance effect showed a strong linear trend $[\operatorname{tg}(30)=5.42$, $\mathrm{p}<.001]$ and a marginally significant quadratic trend $[\mathrm{t}(30)=1.73, \mathrm{p}<.05]$.

Figure 4 shows the number of errors out of a possible seven as a function of direction and distance. For each direction, the greatest number of errors occurred adjacent to the forward-backward boundary. An ANOVA was conducted for number of errors with a design identical to that for response time. Sex was not significant as a main effect or in interaction. Distance $[F(2,30)=7.68, p<.01]$ and direction $[F(1,15)=6.09, p<.05]$ were both significant, but their interaction was not. Planned comparisons revealed a significant linear trend for distance $[\mathrm{t}(30)=$ $2.54, p<.01]$, but the quadratic trend was not significant.

Mean method importance scores for the six methods were: (1) 3.06 , (2) 4.00 , (3) 2.06 , (4) 3.18, (5) 1.06, and (6) 2.35. In a repeated measures ANOVA with method as a within-subject factor, method was significant $[F(5,80)=4.13, p<.01]$. A planned comparison pitted "picturing the days"

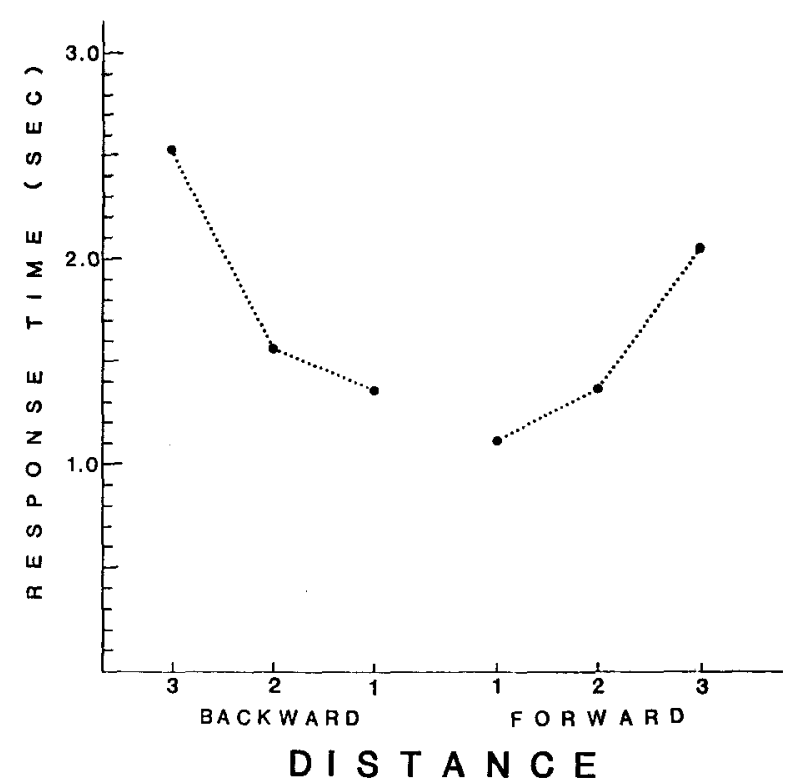

Figure 3. Mean response time (in seconds) for each forward and backward distance for the day-of-the-week task (Experiment 2). 


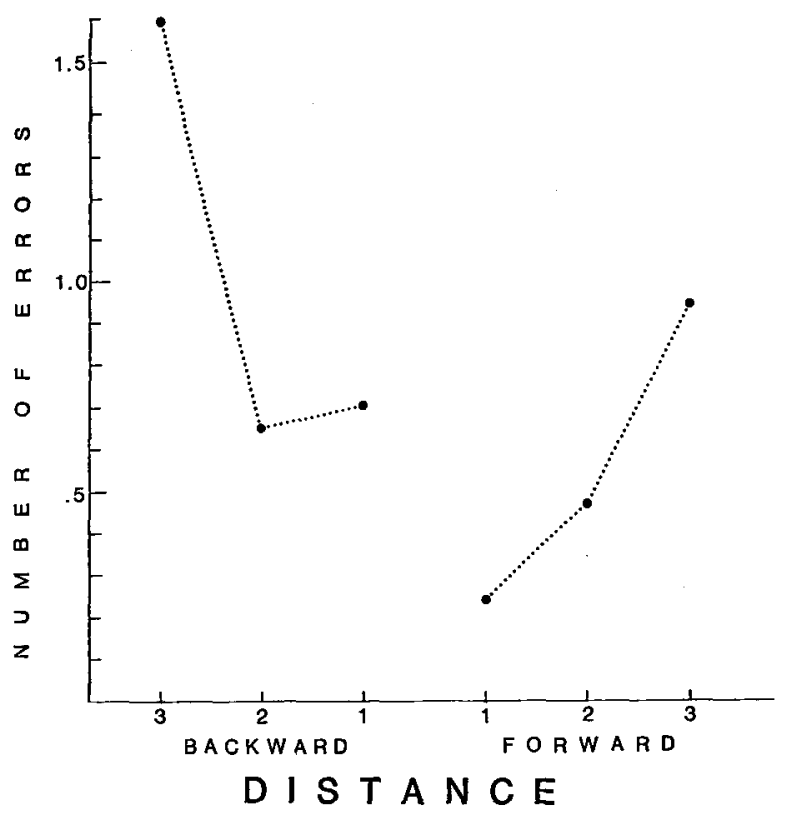

Figure 4. Mean number of errors out of a posalble 7 for each forward and backward diatance for the day-of-the-week task (Experiment 2).

against the other methods. The comparison was significant $[\mathrm{t}(80)=3.01, \mathrm{p}<.01]$.

The proximity effects for response time and accuracy are consistent with the analog model and serve to extend the findings of Experiment 1 to a second content. Experiment 2 also shows that the proximity effects are not restricted to the particular methods used in the original study.

The linear effect is inconsistent with a hybrid arithmetic-decision rule model. In addition, it is notable that numerical methods were virtually never reported on this task. While addition or subtraction of month numbers may be a useful way to determine the boundary in the month task, subjects do not appear to use a similar method in the present task. This may be because day-number correspondence schemes are less common than mouth-number schemes or because the boundary can be determined more easily by other methods for sets with only seven elements. The quadratic effect was relatively weak in Experiment 2, but the approximate symmetry of the forward and backward response curves and the absence of a distance $x$ direction interaction rule out the serial activation model.

The significant direction effects are not predicted by the analog model. As for the month task, the effect may be due in part to the automatic activation of a verbal list representation for close forward pairs. However, the more than 400-msec difference between the forward 3 and backward 3 means and the absence of a distance $\times$ direction interaction show that this is not a complete explanation. Friedman (1983) found direction effects for tasks in which subjects judged the relative order of two months in either backward or forward directions from a third month. I suggested that, outside the laboratory, the subjects might use forward-order operations more frequently and that this might result in a bias akin to the leftto-right scanning bias of readers of English. The design of the present study is inadequate to evaluate this interpretation of the direction effect.

\section{GENERAL DISCUSSION}

The experiments reported here demonstrate proximity effects for a cyclic relationship using two familiar contents. Analog models of the SDE for linear relationships are easily extended to cyclic relationships and imply the type of proximity effects found in this study. The particular analog model used here accurately predicted linear and quadratic increases in difficulty as separations approached half of a cycle. In addition, the patterns of response times and errors for the month task were quite similar to those of the spatial task, lending further plausibility to the analog interpretation. In spite of the general success of the present analog model, further research would be needed to specify in detail the processes used in these tasks. It is not clear, for example, whether the separation effect is due to the additional time needed to precisely delineate the boundary when the second month is close to it or to the difficulty of discriminating the second month from the boundary, apart from the time taken to activate them.

Semantic models of the SDE are not easily extended to cyclic relationships, because they depend upon bipolar or categorical descriptions of the items that are available in natural language. These sorts of descriptions do not contain sufficient information to determine the relative cyclic order of items. If this claim is correct, then the proximity effects shown in the present study would seem to weaken the status of existing semantic models as general accounts of proximity effects in comparative judgments.

Two alternative models were evaluated, and neither received support. A self-terminating forward serial search model was inconsistent with the approximate symmetry of the forward and backward curves and with the quadratic distance effects. However, there was some indication that a verbal list process was automatically activated when the second month closely followed the first. A hybrid arithmeticdecision rule model failed to predict the linear distance effect, and regression analyses provided no support for subtraction operations. Another difficulty for an arithmetic model is that the month task produced a pattern of results similar to that for the spatial task to which numerical solutions seem highly unlikely.

In addition to its relevance to models of symbolic comparisons, the present study adds to our limited information about knowledge of time systems. 
Taken together with previous studies (Fairbanks, 1969; Friedman, 1983; Seymour, 1980a, 1980b), it shows that adults can make many types of inferences about the order of months and days of the week. Although some of these require localization along a bipolar continuum, others involve the determination of precise distances and still others involve relationships of cyclic proximity. It seems unlikely that any single processing model can account for performance on all of the tasks that have been employed. However, the two-process model described by Friedman (1983) appears to offer a foundation for explaining performance on many of them. Tasks that involve the determination of exact distances appear to invoke a verbal-list process, whereas tasks that require judgments of relative proximity, such as those in the present study, appear to invoke an image process.

\section{REFERENCES}

Banks, W. P. (1977). Encoding and processing of symbolic information in comparative judgments. In G. H. Bower (Ed.), The psychology of learning and motivation (Vol. 11). New York: Academic Press.

Banks, W. P., Fujil, M., \& Kayra-Stuart, F. (1976). Semantic congruity effects in comparative judgments of magnitudes of digits. Journal of Experimental Psychology: Human Perception and Performance, 2, 435-447.

Fairbanks, B. A. (1969). Experiments on the temporal aspects of number perception (Doctoral dissertation, University of Arizona, 1969). Dissertation Abstracts International, 30, 403B. (University Microfilms No. 69-10, 052)

Friedman, W. J. (1982). Conventional time concepts and children's structuring of time. In W. J. Friedman (Ed.), The developmental psychology of time. New York: Academic Press.

Friedman, W. J. (1983). Image and verbal processes in reasoning about the months of the year. Journal of Experimental Psy. chology: Learning, Memory, and Cognition, 9, 650-666.

Hamilton, J. M. E., \& SAnford, A. J. (1978). The symbolic distance effect for alphabetic order judgments: A subjective report and reaction time analysis. Quarterly Journal of Experimental Psychology, 30, 33-43.

Holyonk, K. (1978). Comparative judgments with numerical reference points. Cognitive Psychology, 10, 203-243.

KossLYN, S. M. (1981). The medium and the message in mental imagery: A theory. Psychological Review, 88, 45-66.

Lovelace, E. A., Powell, C. M., \& Brooks, R. J. (1973). Alphabetic position effects in covert and overt alphabetic recitation times. Journal of Experimental Psychology, 99, 405-408.

MOYER, R. S. (1973). Comparing objects in memory: Evidence suggesting an internal psychophysics. Perception \& Psychophysics, 13, 180-184.

Parvio, A. (1975). Perceptual comparisons through the mind's eye. Memory \& Cognition, 3, 635-647.

PAivio, A. (1978). Dual coding: Theoretical issues and empirical evidence. In J. C. Scandura \& C. J. Brainerd (Eds.), Structural/ process models of complex human behavior. Alphen aan den Rijn, The Netherlands: Sijthoff \& Noordhoff.

SEYMOUn, P. H. K. (1980a). Internal representation of the months: An experimental analysis of spatial forms. Psychological Research, 42, 255-273.

Seymour, P. H. K. (1980b). Semantic and structural coding of the months. British Journal of Psychology, 71, 371-393.

Shepard, R. N., \& Podgorny, P. (1978). Cognitive processes that resemble perceptual processes. In W. Estes (Ed.), Handbook of learning and cognitive processes: Vol. 5. Human information processing. Hillsdale, NJ: Erlbaum.

Shoben, E. J., Cech, C. G., \& Schwanenflugel, P. J. (1983). The role of subtractions and comparisons in comparative judgments involving numerical reference points. Journal of Experimental Psychology: Human Perception \& Performance, 9, 226-241.

Te Linde, J., \& Paivio, A. (1979). Symbolic comparison of color similarity. Memory \& Cognition, 7, 141-148.

Weber, R. J., Cross, M., \& Carlton, M. (1968). Searching circular sequences. Journal of Experimental Psychology, 78, 588-592.

Wickelaren, W. A. (1979). Cognitive psychology. Englewood Cliffs, NJ: Prentice-Hall.

(Manuscript received November 25, 1983; revision accepted for publication January 5, 1984.) 